Article

\title{
Synthesis and Anticancer Activities of Novel Guanylhydrazone and Aminoguanidine Tetrahydropyran Derivatives
}

\author{
Fábio Pedrosa Lins Silva ${ }^{1}$, Bruna Braga Dantas ${ }^{2}$, Gláucia Veríssimo Faheina Martins ${ }^{2}$, \\ Demétrius Antônio Machado de Araújo ${ }^{2}$ and Mário Luiz Araújo de Almeida Vasconcellos 1,* \\ 1 Departamento de Química, Campus I, Laboratório de Síntese Orgânica Medicinal da Paraíba (LASOM-PB), \\ Universidade Federal da Paraíba, João Pessoa, CEP:58051-900, Paraíba, Brazil; pedrosalinssilva@gmail.com \\ 2 Departamento de Biotecnologia, Campus I, Laboratório de Biotecnologia Celular e Molecular, \\ Universidade Federal da Paraíba, João Pessoa, CEP:58051-900, Paraíba, Brazil; \\ brunabdantas@gmail.com (B.B.D.); glauciafaheina@yahoo.com.br (G.V.F.M.); \\ demetrius@cbiotec.ufpb.br (D.A.M.A.) \\ * Correspondence: mlaav@quimica.ufpb.br; Tel.: +55-83-3216-7589
}

Academic Editor: Derek J. McPhee

Received: 28 March 2016; Accepted: 11 May 2016; Published: 21 June 2016

\begin{abstract}
In this paper we present the convenient syntheses of six new guanylhydrazone and aminoguanidine tetrahydropyran derivatives 2-7. The guanylhydrazone 2, 3 and 4 were prepared in $100 \%$ yield, starting from corresponding aromatic ketones $\mathbf{8 a}-\mathbf{c}$ and aminoguanidine hydrochloride accessed by microwave irradiation. The aminoguanidine 5, 6 and 7 were prepared by reduction of guanylhydrazone 2-4 with sodium cyanoborohydride (94\% yield of 5, and 100\% yield of 6 and 7). The aromatic ketones $\mathbf{8 a}-\mathbf{c}$ were prepared from the Barbier reaction followed by the Prins cyclization reaction (two steps, 63\%-65\% and 95\%-98\%). Cytotoxicity studies have demonstrated the effects of compounds 2-7 in various cancer and normal cell lines. That way, we showed that these compounds decreased cell viabilities in a micromolar range, and from all the compounds tested we can state that, at least, compound 3 can be considered a promising molecule for target-directed drug design.
\end{abstract}

Keywords: Prins cyclization reaction; tetraydropyran derivatives; anticancer; guanylhydrazone; aminoguanidine

\section{Introduction}

Despite the availability of improved drugs, including targeted cancer therapies, cancer is still one of the leading causes of mortality worldwide. It is estimated to have accounted for 8.2 million deaths (around 13\% of all deaths) in 2007, and $~ 1.4$ million new cancer cases and $~ 566,000$ deaths from cancer occurred in the United States in 2008 [1-4]. Additionally, a 70\% increase in new cases of cancer is expected over the next two decades. Therefore, new, more effective treatment strategies are required to address this issue. A substantial number of new antineoplastic agents have been discovered. Considerable insight has been gained into the mechanisms by which many of these compounds affect cellular growth and this knowledge has been used in the design of new chemotherapeutic drugs [5].

In recent years, several compounds based on the tetrahydropyran moiety have been described as presenting high anticancer activities [6-11]. Substituted tetrahydropyranyl moieties are extensively distributed in the natural products' structures that present a large pharmacological profile [12]. There are many synthetic methodologies to prepare this moiety, i.e., the Prins cyclization reaction and others. In 2014, Ahmed et al. described several 2,4,6-diaryltetraydropyran scaffolds that possess immense importance with antiproliferative activity against human cancer cell lines (Figure 1) [13]. 


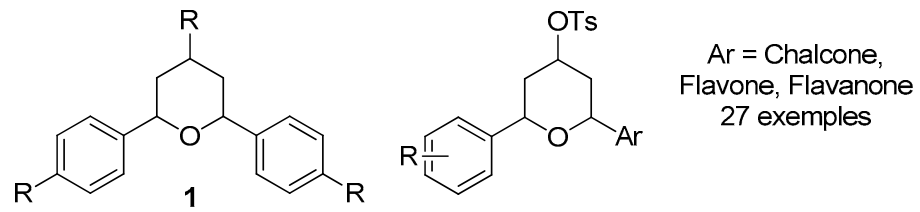

Figure 1. The compound 2,4,6-Diaryltetraydropyran has antiproliferative activity against human cancer cell lines.

In connection to our interest in the stereoselective synthesis of tetraydropyran derivatives using the Prins cyclization reaction [14-18], and considering that guanylhydrazone [19] and aminoguanidine groups $[20,21]$ potentiate the anticancer activity of some drugs, and these nitrogenated bases could be considered pharmacophoric points, we present in this article the syntheses of six novel tetrahydropyranyl guanylhydrazone and aminoguanidine 2-7 (Figure 2). That way, we performed in vitro evaluation for anticancer activity against cancer cell lines, such as the chronic myeloid leukemia (K562), human acute myeloid leukemia (HL-60), human breast adenocarcinoma (MCF-7), human colon adenocarcinoma (HT-29), and L929 (murine fibroblast) and the human peripheral blood of patients with chronic myeloid leukemia (PBMC/CML) cells. Normal cell lines, L929 (murine fibroblast) cells and human peripheral blood cells (PBMC) were also evaluated.<smiles>N=C(N)NN=C1CC(c2ccccc2)OC(c2ccccc2)C1</smiles>

2<smiles>N=C(N)NNC1CC(c2ccccc2)OC(c2ccccc2)C1</smiles>

5<smiles>N=C(N)NN=C1CC(c2ccc(F)cc2)OC(c2ccc(F)cc2)C1</smiles><smiles>N=C(N)NNC1CC(c2ccc(F)cc2)OC(c2ccc(F)cc2)C1</smiles><smiles>N=C(N)NN=C1CC(c2ccc3ccccc3c2)OC(c2ccc3ccccc3c2)C1</smiles><smiles>N=C(N)NN[C@H]1C[C@@H](c2ccc3ccccc3c2)O[C@@H](c2ccc3ccccc3c2)C1</smiles>

Figure 2. Compounds synthesized and evaluated against tumor cells line in this work.

It is important to note that compounds $\mathbf{2 - 7}$ are Meso compounds which greatly simplifies these syntheses, since only relative configuration (cis \trans) must be controlled [12].

\section{Results and Discussion}

\subsection{Chemistry}

Our experimental work starts with the preparation in good yield of homoallylic alcohols shown in Scheme 1 (step i) from the Barbier reaction [22] between the corresponding aldehydes (benzaldehyde, 4-fluorobenzaldehyde and 2-naphthaldehyde) with allyl bromide in the presence of stannous chloride (96\%-98\% yields). In step ii the corresponding tetrahydropyran rings of $\mathbf{1 a}-\mathbf{c}$ are prepared with control of relative configuration (2,4,6-cis) from the Prins cyclization reaction [12], followed by hydrolysis of the acetyl intermediate with potassium carbonate in methanol ( $60 \%-63 \%$ yields). The cis stereoselectivity of these reactions is anticipated based on the proposed mechanisms 4 for these reactions and determined by bi-dimensional spectroscopic study (NOESY spectra, see in Supplementary Materials) and by X-ray crystallography of $\mathbf{1}$ [23]. The oxidation of tetrahydropyran alcohols to the corresponding ketones $\mathbf{8 a - c}$ (step iii, Scheme 1) was performed in high yields by using PCC in dichloromethane as a solvent 
(95\%-98\% yields). The guanylhydrazone $2-\mathbf{4}$ (Figure 1) were prepared in quantitative yields (100\% yields) by reacting ketones $\mathbf{8} \mathbf{a}-\mathbf{c}$ with aminoguanidine hydrochloride and ethanol as a solvent (no catalysts were used) promoted by $5 \mathrm{~min}$ of microwave irradiation $(250 \mathrm{~W})$ at $100{ }^{\circ} \mathrm{C}$ (step iv). Finally, the reductions of aminoguanidine $2-\mathbf{4}$ were performed in very high yields ( $94 \%-100 \%$ yields) by reacting 2-4 with sodium cyanoborohydride in ethanol as a solvent at room temperature (step $\mathrm{v}$, Scheme 1). The relative configurations of $\mathrm{C} 4$ of aminoguanidine $5-\mathbf{7}$ were determined by ${ }^{1} \mathrm{H}-\mathrm{NMR}$ spectroscopic studies (NOESY spectrum, see in Supplementary Materials).

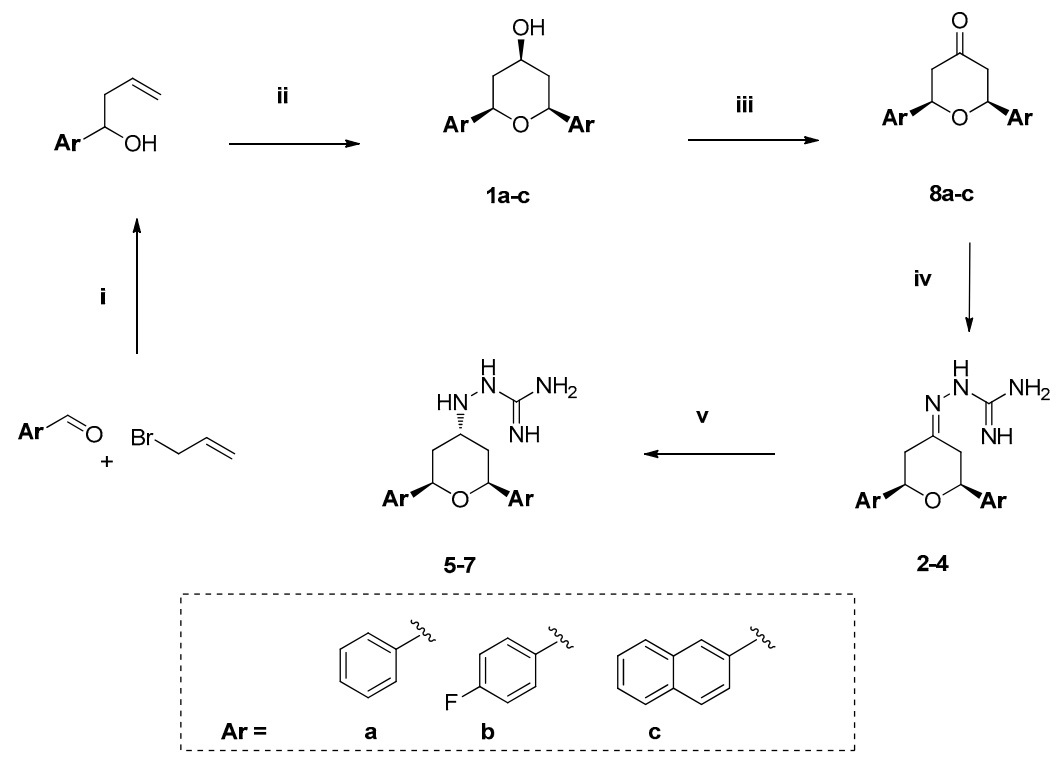

Scheme 1. Synthesis of guanylhydrazone 2-4 and aminoguanidine 5-7. (i) $\mathrm{SnCl}_{2}, \mathrm{KI}, \mathrm{H}_{2} \mathrm{O}, \mathrm{NH}_{4} \mathrm{Cl}$, $0{ }^{\circ} \mathrm{C}, 2$ h, r.t., $96 \%-98 \%$ yields; (ii) 1- $\mathrm{PhH}, \mathrm{AcOH}, \mathrm{BF}_{3} \cdot \mathrm{Et}_{2} \mathrm{O}, 0{ }^{\circ} \mathrm{C}, 3$ h, r.t.; $2-\mathrm{K}_{2} \mathrm{CO}_{3}, \mathrm{MeOH}, 30$ min, r.t., $60 \%-63 \%$ yields; (iii) $\mathrm{PCC}, \mathrm{CH}_{2} \mathrm{Cl}_{2}$, r.t., $2.5 \mathrm{~h}, 95 \%-98 \%$ yields; (iv) aminoguanidine hydrochloride, $\mathrm{EtOH}$, microwave, $100{ }^{\circ} \mathrm{C}, 5 \mathrm{~min}, 100 \%$ yields; (v) $\mathrm{NaCNBH}_{3}, 0^{\circ} \mathrm{C}, 2$ h, r.t., $94 \%-100 \%$ yields.

\subsection{Biology}

The anticancer drug discovery screening has been designed to distinguish between broad-spectrum anticancer compounds and cancer-selective agents [24]. The anticancer activities of 2-7 were investigated against different types of cancer cells and non-cancer cells using MTT assay. Each cell line was incubated with different concentrations $\left(3-100 \mu \mathrm{mol} \cdot \mathrm{L}^{-1}\right)$ for each compound and this was used to create a concentration-response relationship curve. The response parameter $\left(\mathrm{IC}_{50}\right)$ was calculated for each cell line. The $\mathrm{IC}_{50}$ value corresponds to the compound's concentration causing $50 \%$ of reduction viability at the end of the incubation period ( 24 or $72 \mathrm{~h}$ ). Compounds 2-7 indicated potency anticancer activity against K562, HL-60, MCF-7, HT-29 and PBMC/CML cell lines. The analogues showed a broad-spectrum anticancer activity independent of the incubation period (Tables 1 and 2).

The effects of compounds 3 and 7 , which were the most promissory substances, had similar action on K562 and PBMC/CML cells. Moreover, those cells showed to be the most sensitive cells. It is important because those types of chronic myeloid leukemia have expressed the BCR-ABL gene, which stimulates cell growth, and it is highly resistant to anticancer therapy $[25,26]$. Therefore, our result can address an association with a possible clinical application.

In this work, etoposide has been used as the reference substance, because this compound is known is a standard drug used in the treatment of a wide spectrum of solid tumors, lymphomas and leukemias. It is a topoactive drug which inhibits topoisomerase II-DNA cleavable complex, resulting in DNA damage, and often results in cell death apoptosis [27,28]. 
The results have demonstrated that DNA is vulnerable to damage after treatment with compounds 3 and 7, similar to therapeutics such as etoposide. Many anticancer drugs exert their effect on the cell cycle which is known as being cell-cycle-specific. The compounds 3 and 7 induced cell-cycle arrest (Supplement Figures S1 and S2) in the G1 phase, corresponding to IC 50 in 24 h, as well as an increase of cells in the sub-G1 phase, representing sub diploid content that corresponds to apoptotic cells with fragmented DNA or condensed chromatin [29]. This event demonstrates that the addition of the compound inhibits the cell cycle and induces cell death by apoptosis, probably because these cells were unable to repair the damage caused by molecules [30].

Our results also showed that the different substituents added around the tetrahydropyran ring were critical to the motifs' efficacy and our cytotoxic data showed that compounds 2, 3 and 4 are, at least, two-fold more potent than compounds 5, 6 and 7 (Tables 1 and 2). The analogs 3 and 6 become more powerful than 2 and 5, probably due to the addition of the fluor atom at position 4 in the aromatic ring. Compounds 4 and 7 were even more potent than the other analogs, probably due to the presence of two naphthyl rings, which alter the molecular properties, i.e., lipophilicity, among others. Other studies corroborate with our results, demonstrating increased activity of tetrahydropyran derivates with a further lipophilicity group [31].

The six compounds 2-7 demonstrated potent anticancer activity. Moreover, compound 3 showed the most promising effects due to both its powerful cytotoxic activity in K562 cells and being the most selective compound tested [32].

Table 1. Inhibitory effect of compounds and etoposide on cell growth of human cancer and normal cell lines after $24 \mathrm{~h}$ of incubation.

\begin{tabular}{cccccccc}
\hline Compound & HL-60 & K562 & HT-29 & MCF-7 & LMC & L929 & PBMC \\
\hline $\mathbf{2}$ & $16.0 \pm 4.6$ & $13.6 \pm 4.5$ & $15.9 \pm 3.7$ & $52.0 \pm 4.6$ & $\mathrm{nt}$ & $18.1 \pm 5.0$ & $19.4 \pm 3.2$ \\
$\mathbf{3}$ & $12.5 \pm 4.5$ & $8.9 \pm 4.0$ & $11.2 \pm 4.0$ & $31.7 \pm 4.7$ & $7.5 \pm 2.4$ & $20.0 \pm 4.2$ & $14.3 \pm 3.2$ \\
$\mathbf{4}$ & $9.2 \pm 4.6$ & $7.5 \pm 4.9$ & $7.3 \pm 4.7$ & $16.7 \pm 5.2$ & $\mathrm{nt}$ & $10.8 \pm 5.5$ & $5.9 \pm 3.3$ \\
$\mathbf{5}$ & $42.0 \pm 4.4$ & $44.7 \pm 4.8$ & $46.9 \pm 4.2$ & $>100$ & $\mathrm{nt}$ & $65.8 \pm 4.7$ & $51.5 \pm 3.0$ \\
$\mathbf{6}$ & $85.4 \pm 5.2$ & $58.8 \pm 4.1$ & $59.5 \pm 4.3$ & $126.9 \pm 4.3$ & $\mathrm{nt}$ & $72.2 \pm 3.9$ & $72.7 \pm 3.4$ \\
$\mathbf{7}$ & $35.3 \pm 5.2$ & $15.5 \pm 5.1$ & $25.8 \pm 4.8$ & $43.3 \pm 5.8$ & $14.8 \pm 2.4$ & $39.7 \pm 5.5$ & $20.8 \pm 3.5$ \\
Etoposide & $5.8 \pm 1.1$ & $>50$ & $>100$ & $>100$ & $\mathrm{nt}$ & $\mathrm{nt}$ & $>100$ \\
\hline
\end{tabular}

Note: nt (not tested); PBMC, peripheral blood mononuclear cell; SEM, standard error of the mean. HL-60: leukemia myeloid acute, K562: leukemia myeloid chronic, MCF-7: breast adenocarcinoma cell line, HT-29: colon adenocarcinoma cell line and PBMC were incubated with compounds for $24 \mathrm{~h}$ separately. The cell viability was determined by MTT assay. Results are displayed as $\mathrm{IC}_{50}$ in micromolar values and expressed as the mean \pm SEM, obtained from at least three independent experiments in triplicate.

Table 2. Inhibitory effect of compounds and etoposide on cell growth of human cancer and normal cell lines after $72 \mathrm{~h}$ of incubation.

\begin{tabular}{cccccccc}
\hline Compound & HL-60 & K562 & HT-29 & MCF-7 & PBMCLMC & L929 & PBMC \\
\hline $\mathbf{2}$ & $14.4 \pm 5.2$ & $31.0 \pm 4.5$ & $9.6 \pm 4.7$ & $34.8 \pm 3.8$ & $\mathrm{nt}$ & $23.9 \pm 4.0$ & $4.7 \pm 2.9$ \\
$\mathbf{3}$ & $14.9 \pm 5.4$ & $29.0 \pm 4.9$ & $6.0 \pm 4.6$ & $20.8 \pm 4.0$ & $14.6 \pm 3.4$ & $17.2 \pm 4.7$ & $6.7 \pm 3.1$ \\
$\mathbf{4}$ & $6.9 \pm 4.9$ & $19.2 \pm 5.3$ & $3.4 \pm 4.7$ & $13.7 \pm 5.0$ & $\mathrm{nt}$ & $3.9 \pm 3.8$ & $2.2 \pm 2.9$ \\
$\mathbf{5}$ & $41.4 \pm 5.8$ & $71.4 \pm 5.0$ & $19.9 \pm 3.9$ & $47.2 \pm 3.5$ & $\mathrm{nt}$ & $46.9 \pm 4.4$ & $11.4 \pm 2.7$ \\
$\mathbf{6}$ & $66.5 \pm 5.3$ & $>100$ & $36.9 \pm 3.3$ & $42.9 \pm 4.2$ & $\mathrm{nt}$ & $79.4 \pm 3.8$ & $12.5 \pm 2.9$ \\
$\mathbf{7}$ & $25.4 \pm 5.3$ & $43.6 \pm 5.6$ & $11.8 \pm 4.3$ & $24.14 \pm 3.5$ & $18.7 \pm 2.7$ & $16.88 \pm 4.1$ & $7.5 \pm 3.4$ \\
\hline
\end{tabular}

Note: nt (not tested); PBMC, peripheral blood mononuclear cell; SEM, standard error of the mean. HL-60: leukemia myeloid acute, K562: leukemia myeloid chronic, MCF-7: breast adenocarcinoma cell line, HT-29: colon adenocarcinoma cell line and PBMC were incubated with compounds for $24 \mathrm{~h}$ separately. The cell viability was determined by MTT assay. Results are displayed as $\mathrm{IC}_{50}$ in micromolar values and expressed as the mean \pm SEM, obtained from at least three independent experiments in triplicate. 


\section{Materials and Methods}

\subsection{Chemistry}

\subsubsection{General Methods}

All commercially available reagents were purchased from Aldrich and used without further purification. Reactions were monitored by thin-layer chromatography (TLC) using Silica gel 60 UV254 Macherey-Nagel pre-coated silica gel plates; detection was by means of a UV lamp and revelation to vanillin. Flash column chromatography was performed on 300-400 mesh silica gel. Organic layers were dried over anhydrous $\mathrm{MgSO}_{4}$ or $\mathrm{Na}_{2} \mathrm{SO}_{4}$ prior to evaporation on a rotary evaporator. The reactions performed under microwave irradiation were performed in a microwave reactor model CEM Discover System-Benchmate (CEM Corporation, 3100 Smith Farm Road Matthews, NC. USA, 28104) quipped with a system for continuous irradiation with programmable $\mu \mathrm{W}$ power in the range from 0 to $300 \mathrm{~W}$, with temperature monitored by infrared sensor. ${ }^{1} \mathrm{H}$ - and ${ }^{13} \mathrm{C}-\mathrm{NMR}$ spectra were obtained by using a Varian Mercury Spectra AC 200 (200 MHz for ${ }^{1} \mathrm{H}$ and $50 \mathrm{M}$ for ${ }^{13} \mathrm{C}$ ) (Varian, Palo Alto, CA, USA) or in $\mathrm{CDCl}_{3}$, DMSO or $\mathrm{CD}_{3} \mathrm{OD}$. Spectral patterns are designated as s singlet; d doublet; dd doublet of doublets; ddd double doublet of doublets; $\mathrm{t}$ triplet; $\mathrm{dt}$ double triplet; q quartet; $\mathrm{m}$ multiplet. The chemical shifts $(\delta, \mathrm{ppm})$ were reported in values relative to tetramethylsilane $(0 \mathrm{ppm})$ for ${ }^{1} \mathrm{H}-\mathrm{NMR}$ and were reported in the scale relative to $\mathrm{CDCl}_{3}$ (77.00 ppm), DMSO (39.52 ppm) or $\mathrm{CD}_{3} \mathrm{OD}$ (49.00 ppm) for ${ }^{13} \mathrm{C}-\mathrm{NMR}$ and coupling constants $(J)$ were expressed in. The Fourier Transform Infrared Spectroscopy spectra were obtained using a spectrophotometer IR-Prestige-21 (Shimadzu, Kyoto, Japan). MS data were measured with a Shimadzu GCMS-QP2010 mass spectrometer (Shimadzu). The elementary analyses of unpublished compounds were performed in an analyzer organic elemental CHNS-O, FLASH 2000 of Perkin-Elmer, Waltham, MA, USA).

\subsubsection{General Procedure for Homoallylic Alcohol Synthesis}

The synthesis of homoallylic alcohol was performed through a Barbier reaction. The corresponding aldehyde $(8 \mathrm{mmol})$ was added to water $(40 \mathrm{~mL})$ containing potassium iodide $(24 \mathrm{mmol})$, stannous chloride dehydrate $(12 \mathrm{mmol})$ and allyl bromide $(1 \mathrm{~mL})$. The orange solution turns white by addition of saturated ammonium chloride $(20 \mathrm{~mL})$. The stirring was continued for $2 \mathrm{~h}$ at room temperature. After the end of reaction, the reaction mixture was extracted with $\mathrm{CH}_{2} \mathrm{Cl}_{2}(2 \times 50 \mathrm{~mL})$ gave an organic phase, washed with water, dried with sodium sulfate anhydrous, concentrated under reduced pressure and purified by flash chromatography on silica gel (EtOAc:Hexane as eluent). The products were concentrated under reduced pressure yielding the homoallylic alcohol, respectively [23].

1-Phenylbut-3-en-1-ol. This product was obtained using benzaldehyde. The product was purified by silica gel column chromatography using EtOAc/hexane (1:9) as eluent, resulting in 97\% yield. IR (KBr) $v / \mathrm{cm}^{-1} 3371,3070,3028,2904,1639,1492,1049,995,914,756,702 ;{ }^{1} \mathrm{H}-\mathrm{NMR}\left(200 \mathrm{MHz}, \mathrm{CD}_{3} \mathrm{OD}\right) \delta 7.33$ $(\mathrm{m}, 5 \mathrm{H}), 5.83(\mathrm{~m}, 1 \mathrm{H}), 5.18(\mathrm{~m}, 2 \mathrm{H}), 4.74(\mathrm{t}, 1 \mathrm{H}, \mathrm{J}=6.0 \mathrm{~Hz}), 2.53(\mathrm{~m}, 3 \mathrm{H}) ;{ }^{13} \mathrm{C}-\mathrm{NMR}\left(50 \mathrm{MHz} \mathrm{CDCl}_{3}\right)$ $\delta 144.57,135.18,129.09,128.22,126.54,119.02,74.03,44.49$.

1-(4-Fluorophenyl)but-3-en-1-ol. This product was obtained using 4-fluorobenzaldehyde. The product was purified by silica gel column chromatography using EtOAc/hexane (3:7) as eluent, resulting in $96 \%$ yield. IR (KBr) v/ $\mathrm{cm}^{-1} 3383,3078,2981,2908,1604,11508,1049,995,914,837 ;{ }^{1} \mathrm{H}-\mathrm{NMR}(200 \mathrm{MHz}$, $\left.\mathrm{CDCl}_{3}\right) \delta 7.41(\mathrm{~m}, 2 \mathrm{H}), 7.14(\mathrm{~m}, 2 \mathrm{H}), 5.88(\mathrm{~m}, 1 \mathrm{H}), 5.25(\mathrm{~m}, 2 \mathrm{H}), 4.80(\mathrm{t}, 1 \mathrm{H}, J=6.0 \mathrm{~Hz}), 2.57(\mathrm{~m}, 3 \mathrm{H})$; ${ }^{13} \mathrm{C}-\mathrm{NMR}\left(50 \mathrm{MHz}, \mathrm{CDCl}_{3}\right) \delta 165.29,160.42,140.31,140.25,134.96,134.88,128.29,128.13,119.36,116.13$, $115.71,73.45,73.33,44.62$.

1-(Naphthalen-2-yl)but-3-en-1-ol. This product was obtained using 2-naphthaldehyde. The product was purified by silica gel column chromatography using EtOAc/hexane (1:9) as eluent, resulting in $98 \%$ yield. IR (KBr) $v / \mathrm{cm}^{-1} 3278,3051,2947,2854,1600,1508,1060,991,952,821,748 ;{ }^{1} \mathrm{H}-\mathrm{NMR}(200 \mathrm{MHz}$, 
$\left.\mathrm{CDCl}_{3}\right) \delta 7.67(\mathrm{~m}, 7 \mathrm{H}), 5.84(\mathrm{~m}, 1 \mathrm{H}), 5.18(\mathrm{~m}, 2 \mathrm{H}), 4.90(\mathrm{t}, 1 \mathrm{H}, J=6.0 \mathrm{~Hz}), 2.60(\mathrm{~m}, 2 \mathrm{H}), 2.34(\mathrm{~s}, 1 \mathrm{H})$;

${ }^{13} \mathrm{C}-\mathrm{NMR}\left(50 \mathrm{MHz} \mathrm{CDCl}_{3}\right) \delta 141.98,135.11,133.98,133.67,128.94,128.70,128.42,126.86,126.55,125.26$, $124.75,119.23,74.19,44.45$.

\subsubsection{General Procedure for Synthesis of 4-Hydroxy-2,6-diaryl-tetrahydropyran}

A round bottom flask equipped with a magnetic stir bar was charged with $0.81 \mathrm{~mL}$ of acetic acid, $7 \mathrm{~mL}$ of benzene, $4.5 \mathrm{mmol}$ of homoallylic alcohol and $9 \mathrm{mmol}$ of aldehyde. The mixture was cooled to $0{ }^{\circ} \mathrm{C}$ and after slow addition of $1.2 \mathrm{~mL}$ of boron trifluoride etherate was stirred in this temperature for $3 \mathrm{~h}$. To the reaction media was added saturated $\mathrm{NaHCO}_{3}$ in water $(10 \mathrm{~mL})$ followed extraction with EtOAc $(3 \times 10 \mathrm{~mL})$. The combined organic phase are washed with brine $(3 \times 10 \mathrm{~mL})$, dried over $\mathrm{Na}_{2} \mathrm{SO}_{4}$, filtered and concentrated under vacuum. The acetylated thus obtained in this reaction was dissolved in methanol $(10 \mathrm{~mL})$ and stirred over potassium carbonate $(500 \mathrm{mg})$ for $0.5 \mathrm{~h}$. Then methanol was removed under reduced pressure and water $(20 \mathrm{~mL})$ was added. The mixture was extracted with EtOAc $(2 \times 20 \mathrm{~mL})$ and the combined organic layers were dried over anhydrous $\mathrm{Na}_{2} \mathrm{SO}_{4}$ and the solvent was removed under reduced pressure. The resulting crude product was purified by column chromatography on silica gel to give compound 4-Hydroxy-2,6-disubstituted tetrahydropyran [23].

2,6-Diphenyl-tetrahydro-2H-pyran-4-ol (1a). This product was obtained using $4.5 \mathrm{mmol}$ of 1-phenylbut3-en-1-ol and $9 \mathrm{mmol}$ of benzaldehyde. The product was purified by silica gel column chromatography using EtOAc/hexane (1:3) as eluent, resulting in 60\% yield. IR (KBr) $v / \mathrm{cm}^{-1} 3263,3032,2947,2858$, 1604, 1496, 1319, 1060, 752, 694; ${ }^{1} \mathrm{H}-\mathrm{NMR}\left(200 \mathrm{MHz}, \mathrm{CDCl}_{3}\right) \delta 7.37(\mathrm{~m}, 10 \mathrm{H}), 4.57(\mathrm{~d}, 2 \mathrm{H}, \mathrm{J}=12.0 \mathrm{~Hz})$, $4.10(\mathrm{~m}, 1 \mathrm{H}), 2.27(\mathrm{dd}, 2 \mathrm{H}, J=12.0,4.0 \mathrm{~Hz}), 2.12(\mathrm{~m}, 1 \mathrm{H}), 1.60(\mathrm{q}, 2 \mathrm{H}, J=12.0 \mathrm{~Hz}) ;{ }^{13} \mathrm{C}-\mathrm{NMR}(50 \mathrm{MHz}$, $\left.\mathrm{CDCl}_{3}\right) \delta 142.49,128.85,128.02,126.39,78.32,69.03,43.51$.

2,6-Bis(4-fluorophenyl)-tetrahydro-2H-pyran-4-ol (1b). This product was obtained using $4.5 \mathrm{mmol}$ of 1-(4-fluorophenyl) but-3-en-1-ol and $9 \mathrm{mmol}$ of 4-Fluorobenzaldehyde. The product was purified by silica gel column chromatography using EtOAc/hexane (3:7) as eluent, resulting in 62\% yield. IR (KBr) $v / \mathrm{cm}^{-1} 3329,3051,2943,2854,1604,1512,1230,1068,821 ;{ }^{1} \mathrm{H}-\mathrm{NMR}\left(200 \mathrm{MHz}, \mathrm{CDCl}_{3}\right) \delta 7.33(\mathrm{~m}, 4 \mathrm{H})$, $7.04(\mathrm{~m}, 4 \mathrm{H}), 4.53(\mathrm{~d}, 2 \mathrm{H}, J=12.0 \mathrm{~Hz}), 4.09(\mathrm{~m}, 1 \mathrm{H}), 2.23(\mathrm{~m}, 2 \mathrm{H}), 2.02(\mathrm{~s}, 1 \mathrm{H}), 1.56(\mathrm{dd}, 2 \mathrm{H}, J=14.0$, $12.0 \mathrm{~Hz}) ;{ }^{13} \mathrm{C}-\mathrm{NMR}\left(50 \mathrm{MHz}, \mathrm{CDCl}_{3}\right) \delta 165.36,160.48,138.39,138.33,128.41,128.25,116.18,115.75$, $78.14,78.06,69.09,43.70$.

2,6-Di(naphthalen-2-yl)-tetrahydro-2H-pyran-4-ol (1c). This product was obtained using $4.5 \mathrm{mmol}$ of 1-(naphthalen-2-yl)but-3-en-1-ol and $9 \mathrm{mmol}$ of 2-naphthaldehyde. The product was purified by silica gel column chromatography using EtOAc/hexane $(1: 1)$ as eluent, resulting in $63 \%$ yield. IR $(\mathrm{KBr})$ $v / \mathrm{cm}^{-1} 3278,3020,2947,2854,1600,1508,1357,1060,821,748 ;{ }^{1} \mathrm{H}-\mathrm{NMR}(500 \mathrm{MHz}, \mathrm{DMSO}) \delta 7.92(\mathrm{~m}$, $8 \mathrm{H}), 7.63(\mathrm{dd}, 2 \mathrm{H}, J=10.0,5.0 \mathrm{~Hz}), 7.50(\mathrm{~m}, 4 \mathrm{H}), 4.82(\mathrm{~d}, 2 \mathrm{H}, J=10.0 \mathrm{~Hz}), 4.11(\mathrm{~m}, 1 \mathrm{H}), 3.82(\mathrm{~s}, 1 \mathrm{H}), 2.29$ $(\mathrm{dd}, 2 \mathrm{H}, J=10.0,5.0 \mathrm{~Hz}), 1.58(\mathrm{dd}, 2 \mathrm{H}, J=25.0,10.0 \mathrm{~Hz}) ;{ }^{13} \mathrm{C}-\mathrm{NMR}(125 \mathrm{MHz}, \mathrm{DMSO}) \delta 140.76,133.40$, $132.94,128.41,128.30,128.02,126.59,126.28,125.08,124.64,77.81,67.35,43.63$.

\subsubsection{General Procedure for Oxidation of 4-Hydroxy-2,6-diaryl Tetrahydropyrans}

In a typical reaction, $1 \mathrm{mmol}$ of 4-hydroxy-2,6-disubstituted tetrahydropyran was dissolved in $6 \mathrm{~mL}$ of $\mathrm{CH}_{2} \mathrm{Cl}_{2}$ dry in one round bottom flask and treated with $1 \mathrm{mmol}$ of PCC. The stirring was continued for $2.5 \mathrm{~h}$ at room temperature where the completion of the reaction was monitored by thin-layer chromatography (TLC). The mixture was extracted with $\mathrm{CH}_{2} \mathrm{Cl}_{2}$ and filtered through a short pad of silica gel with hexane and the solvent evaporated to 2,6-disubstituted-tetrahydropyran -4-one. No side products were observed to be formed [23].

2,6-Diphenyl-tetrahydropyran-4-one (8a). This product was obtained using $1 \mathrm{mmol}$ of 2,6-diphenyltetrahydro-2H-pyran-4-ol, resulting in 98\% yield. IR (KBr) v/ $\mathrm{cm}^{-1} 3032,2970,2858,1720,1604,1496$, 1056, 1060, 752, 698; ${ }^{1} \mathrm{H}-\mathrm{NMR}\left(500 \mathrm{MHz}, \mathrm{CDCl}_{3}\right) \delta 7.40(\mathrm{~m}, 10 \mathrm{H}), 4.85(\mathrm{dd}, 2 \mathrm{H}, J=10.0,5.0 \mathrm{~Hz}), 2.73(\mathrm{~m}$, $4 \mathrm{H}) ;{ }^{13} \mathrm{C}-\mathrm{NMR}\left(125 \mathrm{MHz}, \mathrm{CDCl}_{3}\right) \delta 206.39,141.10,128.99,128.43,126.01,79.31,50.06$. 
2,6-Bis(4-fluorophenyl)-tetrahydropyran-4-one (8b). This product was obtained using $1 \mathrm{mmol}$ of 2,6-bis(4-fluorophenyl)-tetrahydro-2H-pyran-4-ol, resulting in 97\% yield. IR (KBr) v/ cm ${ }^{-1} 3070,2970$, 2893,1720, 1608, 1512, 1053, 1138,829; ${ }^{1} \mathrm{H}-\mathrm{NMR}\left(200 \mathrm{MHz}, \mathrm{CDCl}_{3}\right) \delta 7.41(\mathrm{~m}, 4 \mathrm{H}), 7.08$ (m, 4H), 4.82 (dd, $2 \mathrm{H}, J=10.0,4.0 \mathrm{~Hz}), 2.66(\mathrm{~m}, 4 \mathrm{H}) ;{ }^{13} \mathrm{C}-\mathrm{NMR}\left(50 \mathrm{MHz}, \mathrm{CDCl}_{3}\right) \delta 206.25,165.65,160.74,137.14,137.08$, $128.28,128.12,116.54,116.11,79.12,50.35$.

2,6-Di(Naphthalen-2-yl)-tetrahydropyran-4-one (8c). Product 8c was obtained using 1 mmol of 2,6-di(naphthalen-2-yl)-tetrahydro-2H-pyran-4-ol, resulting in 95\% yield. IR (KBr) $v / \mathrm{cm}^{-1} 3051$, 2978, 2897, 1708, 1600, 1508, 1045, 825, 748; ${ }^{1} \mathrm{H}-\mathrm{NMR}\left(200 \mathrm{MHz}, \mathrm{CDCl}_{3}\right) \delta 7.69$ (m, 14H), 5.06 (m, 2H), $2.84(\mathrm{~m}, 4 \mathrm{H}) ;{ }^{13} \mathrm{C}-\mathrm{NMR}\left(50 \mathrm{MHz}, \mathrm{CDCl}_{3}\right) \delta 206.76,138.76,133.95,133.85,129.33,128.83,128.48,127.10$, $126.95,125.38,124.41,79.97,50.42$.

\subsubsection{General Procedure for the Preparation of Guanylhydrazone (2-4)}

The hydrazones were prepared by reaction of ketones with aminoguanidine hydrochloride with the aid of a microwave device. A mixture of 2,6-di-substituted-tetrahydropyran-4-one $(0.5 \mathrm{mmol})$ and aminoguanidine hydrochloride $(0.5 \mathrm{mmol})$ in $1 \mathrm{~mL}$ of ethanol were placed in a glass tube for specific microwave reactor along with a magnetic. A shaker reaction was performed under microwave irradiation to $100{ }^{\circ} \mathrm{C}$ (read monitored by infrared sensor) for $5 \mathrm{~min}$ ("Hold Time") under conditions of a closed vessel. After completion of the reaction, the solvent was evaporated under reduced pressure. This crude product was then subjected to flash column chromatography to yield a solid.

2-(2,6-Diphenyl-2H-pyran-4(3H)-ylidene)hydrazinecarboximidamide (2). This product was obtained using $0.5 \mathrm{mmol}$ of 2,6-diphenyl-tetrahydropyran-4-one (8a). The product was purified by column chromatography on silica gel using methanol/EtOAc (1:9) as eluent, resulting in quantitative yield (100\%). IR (KBr) v/ $\mathrm{cm}^{-1}$ 3348, 3309, 3155, 3062, 3035, 2862, 1674, 1597, 1627, 1492, 1091, 1064, 756, 698; ${ }^{1} \mathrm{H}-\mathrm{NMR}\left(200 \mathrm{MHz}, \mathrm{CD}_{3} \mathrm{OD}\right) \delta 7.36(\mathrm{~m}, 10 \mathrm{H}), 4.68(\mathrm{~m}, 2 \mathrm{H}), 3.17(\mathrm{dt}, 1 \mathrm{H}, J=16.0,2.0 \mathrm{~Hz}), 2.76$ $(\mathrm{dt}, 1 \mathrm{H}, J=14.0,2.0 \mathrm{~Hz}), 2.51(\mathrm{dd}, 1 \mathrm{H}, J=14.0,12.0 \mathrm{~Hz}), 2.27(\mathrm{dd}, J=14.0,12.0 \mathrm{~Hz}, 1 \mathrm{H}) ;{ }^{13} \mathrm{C}-\mathrm{NMR}$ (50 MHz, CD 3 OD) $\delta 159.01,158.57,144.10,130.80,130.24,128.20,82.20,44.71,38.37$; Anal. Calcd for $\mathrm{C}_{18} \mathrm{H}_{21} \mathrm{ClN}_{4} \mathrm{O}$ C, 62.69; H, 6.14; N, 16.25; Found C, 62.27, H, 6.24, N, 16.15.

2-(2,6-Bis(4-fluorophenyl)-2H-pyran-4(3H)-ylidene)hydrazinecarboximidamida (3). This product was obtained using $0.5 \mathrm{mmol}$ of 2,6-bis(4-fluorophenyl)-tetrahydropyran-4-one $(\mathbf{8 b})$. The product was purified by column chromatography on silica gel using methanol/EtOAc (1:9) as eluent, resulting in quantitative yield (100\%). IR (KBr) $v / \mathrm{cm}^{-1} 3390,3352,3271,3066,2958,2850,1670,1604,1627,1508$, 1080, 1056, 825. ${ }^{1} \mathrm{H}-\mathrm{NMR}\left(200 \mathrm{MHz}, \mathrm{CD}_{3} \mathrm{OD}\right) \delta 7.45$ (dd, 4H, J 14.0, 8.0), 7.04 (t, 4H, J = 8.0 Hz), $4.63(\mathrm{t}$, $2 \mathrm{H}, J=12.0 \mathrm{~Hz}), 2.72(\mathrm{~d}, 1 \mathrm{H}, J=16.0 \mathrm{~Hz}), 2.48(\mathrm{dd}, 1 \mathrm{H}, J=14.0,12.0 \mathrm{~Hz}), 2.22(\mathrm{dd}, 1 \mathrm{H}, J=14.0,12.0$ $\mathrm{Hz}), 2.08$ (s, 1H); ${ }^{13} \mathrm{C}-\mathrm{NMR}\left(50 \mathrm{MHz}, \mathrm{CD}_{3} \mathrm{OD}\right) \delta 167.43,162.56,159.86,157.84,140.17,130.29,117.19$, 81.64, 44.65, 38.24; Anal. Calcd for $\mathrm{C}_{18} \mathrm{H}_{19} \mathrm{ClF}_{2} \mathrm{~N}_{4} \mathrm{O}$ C, 56.77; H, 5.03; N, 14.71; Found C, 56.63, H, 5.06, $\mathrm{N}, 14.77$.

2-(2,6-Di(naphthalen-2-yl)-2H-pyran-4(3H)-ylidene)hydrazinecarboximidamida (4). This product was obtained using $0.5 \mathrm{mmol}$ of 2,6-di(naphthalen-2-yl)-tetrahydropyran-4-one (8c). The product was purified by column chromatography on silica gel using methanol/EtOAc (3:7) as eluent, resulting in quantitative yield (100\%). IR (KBr) $v / \mathrm{cm}^{-1} 3423,3340,3155,3055,1672,1600,1625,1508,1078,817,748$. ${ }^{1} \mathrm{H}-\mathrm{NMR}\left(200 \mathrm{MHz}, \mathrm{CD}_{3} \mathrm{OD}\right) \delta 7.87(\mathrm{~m}, 8 \mathrm{H}), 7.60(\mathrm{~m}, 2 \mathrm{H}), 7.43(\mathrm{~m}, 4 \mathrm{H}), 4.78(\mathrm{dd}, 2 \mathrm{H}, J=12.0,2.0 \mathrm{~Hz})$, $2.69(\mathrm{~m}, 4 \mathrm{H}) ;{ }^{13} \mathrm{C}-\mathrm{NMR}\left(50 \mathrm{MHz}, \mathrm{CD}_{3} \mathrm{OD}\right) \delta 158.98,158.34,141.44,135.97,135.83,130.43,130.04,128.55$, 128.41, 127.13, 126.90, 126.35, 82.24, 44.49, 38.26; Anal. Calcd for $\mathrm{C}_{26} \mathrm{H}_{24} \mathrm{~N}_{4} \mathrm{O}$ C, 76.45; H, 5.92; N, 13.72; Found C, 76.55, H, 6.01, N, 13.64 .

\subsubsection{General Procedure for the Preparation of Aminoguanidine (5-7)}

The aminoguanidine were prepared by reduction of guanylhydrazone with sodium cyanoborohydride. The guanylhydrazone $0.5 \mathrm{mmol}$ was added to a dry flask under magnetic stirring, 
followed by addition of $1 \mathrm{~mL}$ of ethanol at $0{ }^{\circ} \mathrm{C}$. Then, $48 \mathrm{mg}$ of sodium cyanoborohydride was slowly added to the flask, and then the mixture remained stirring at room temperature for two hours. After completion of the reaction, the solvent was evaporated under reduced pressure. This crude product was then subjected to flash column chromatography to yield a solid.

2-(2,6-Diphenyltetrahydro-2H-pyran-4-yl)hydrazinecarboximidamida (5). This product was obtained using 0.5 mmol of 2-(2,6-diphenyl-2H-pyran-4-(3H)-ylidene) hydrazinecarboximidamida (2). The product was purified by column chromatography on silica gel using EtOAc as the eluent, resulting in a yield of $94 \%$. IR (KBr) v/ $\mathrm{cm}^{-1} 3568,3448,3062,3032,2862,1674,1631,1631,1492,1126,1064,756,698$; ${ }^{1} \mathrm{H}-\mathrm{NMR}\left(200 \mathrm{MHz}, \mathrm{CD}_{3} \mathrm{OD}\right) \delta 7.33(\mathrm{~m}, 10 \mathrm{H}), 5.40(\mathrm{~s}, 1 \mathrm{H}), 4.60(\mathrm{~m}, 2 \mathrm{H}), 3.10(\mathrm{~d}, 1 \mathrm{H}, J=14.6 \mathrm{~Hz}), 2.33$ $(\mathrm{m}, 2 \mathrm{H}), 1.92(\mathrm{~m}, 2 \mathrm{H}), 1.16(\mathrm{t}, 2 \mathrm{H}, J=8.0 \mathrm{~Hz}) ;{ }^{13} \mathrm{C}-\mathrm{NMR}\left(50 \mathrm{MHz}, \mathrm{CD}_{3} \mathrm{OD}\right) \delta 161.81,159.32,158.24$, 145.58, 145.09, 144.08, 143.32, 130.76, 130.54, 128.19, 82.02, 80.46, 44.71, 42.66, 40.69, 38.21; Anal. Calcd for $\mathrm{C}_{18} \mathrm{H}_{23} \mathrm{ClN}_{4} \mathrm{O}$ C, 62.33; H, 6.68; N, 16.15; Found C, 62.38, H, 6.64, N, 16.19 .

2-(2,6-Bis-(4-fluorophenyl)tetrahydro-2H-pyran-4-yl)hydrazinecarboximidamida (6). This product was obtained using 0.5 mmol of 2-(2,6-bis(4-fluorophenyl)-2H-pyran-4(3H)-ylidene)-hydrazinecarboximidamida (3). The product was purified by column chromatography on silica gel using EtOAc as eluent, resulting in quantitative yield (100\%). IR (KBr) $v / \mathrm{cm}^{-1} 3568,3452,2924,2870,1666,1639,1639,1512,1126$, 1076, 833; ${ }^{1} \mathrm{H}-\mathrm{NMR}\left(200 \mathrm{MHz}, \mathrm{CD}_{3} \mathrm{OD}\right) \delta 7.47(\mathrm{~m}, 4 \mathrm{H}), 7.07(\mathrm{~m}, 4 \mathrm{H}), 4.7(\mathrm{~m}, 2 \mathrm{H}), 3.53(\mathrm{~m}, 2 \mathrm{H}), 1.61(\mathrm{~m}$, $8 \mathrm{H}) ;{ }^{13} \mathrm{C}-\mathrm{NMR}\left(50 \mathrm{MHz}, \mathrm{CD}_{3} \mathrm{OD}\right) \delta 168.48,163.63,159.25,142.88,142.82,142.44,142.38,131.49,118.63$, 118.21, 81.27, 77.61, 41.92, 39.37. Anal. Calcd for $\mathrm{C}_{18} \mathrm{H}_{20} \mathrm{~F}_{2} \mathrm{~N}_{4} \mathrm{O}$ C, 62.42; H, 5.82; N, 16.18; Found C, $62.48, \mathrm{H}, 5.79, \mathrm{~N}, 16.16$.

2-(2,6-Di(naphthalen-2-yl)tetrahydro-2H-pyran-4-yl)hydrazinecarboximidamida (7). This product was obtained using $0.5 \mathrm{mmol}$ of 2-(2,6-di(naphthalen-2-yl)-2H-pyran-4(3H)-ylidene)hydrazinecarboximidamida (4). The product was purified by column chromatography on silica gel using EtOAc as eluent, resulting in quantitative yield (100\%). IR (KBr) $v / \mathrm{cm}^{-1} 3525,3448,3055,2943,1674,1631,1124,1070,819$, 750; ${ }^{1} \mathrm{H}-\mathrm{NMR}\left(200 \mathrm{MHz}, \mathrm{CD}_{3} \mathrm{OD}\right) \delta 7.69(\mathrm{~m}, 14 \mathrm{H}), 4.79(\mathrm{~s}, 2 \mathrm{H}), 2.44(\mathrm{~m}, 5 \mathrm{H}), 1.22(\mathrm{~m}, 2 \mathrm{H}) ;{ }^{13} \mathrm{C}-\mathrm{NMR}$ (50 MHz, CD $\left.{ }_{3} \mathrm{OD}\right) \delta 163.17,160.56,159.60,144.35,143.83,142.76,142.04,137.29,137.14,136.96,131.64$, $131.58,131.23,129.78,129.66,129.40,128.47,128.40,128.20,128.01,127.93,127.67,83.64,82.10,78.42$, 60.92, , 45.87, 41.97, 39.44; Anal. Calcd for $\mathrm{C}_{26} \mathrm{H}_{27} \mathrm{ClN}_{4} \mathrm{O}$ C, 69.87; H, 6.09; N, 12.53; Found C, 69.83, H, $6.12, \mathrm{~N}, 12.64$.

\subsection{Pharmacology}

Fetal bovine serum (FBS) (Cripion, Brazil); Ficoll-Hypaque (GE Healthcare, Uppsala, Sweden); Trypan blue (Inlab, São Paulo, Brazil); trypsin-EDTA and MTT (3-(4,5-Dimethylthiazol-2-yl)2,5-diphenyl tetrazolium bromide) (Amresco, Toronto, ON, Canada); RPMI1640 and DMEM medium was purchased from HIMEDIA (Mumbai, Maharashtra, India) and Penicillin/streptomycin solution was obtained by Sigma-Aldrich (St. Louis, MO, USA).

\subsubsection{Preparation of Stock Solutions}

Preparation of stock solutions of the test compounds were prepared in dimethyl sulfoxide (DMSO) at $20 \mathrm{mmol} \cdot \mathrm{L}^{-1}$, filtered through adequate filters $(0.22 \mu \mathrm{m})$, and diluted for use in the nutrient medium to the relevant working concentrations. For all of the cells used, the nutrient medium was RPMI 1640 or DMEM, according to the cell line tested. We did not verify any DMSO effect, incubating only this dispersing at the highest concentration used in the experiments.

\subsubsection{Cell Culture}

K562, HL-60, MCF-7, HT-29 and L929 cell lines were acquired from Rio de Janeiro Cell Bank (Federal University of Rio de Janeiro, Rio de Janeiro, RJ, Brazil). Leukemia cell lines and MCF-7 cells were maintained in the nutrient RPMI 1640 medium supplemented with $10 \% \mathrm{FBS}, 2 \mathrm{mmol} \cdot \mathrm{L}^{-1}$ 
glutamine, $100 \mathrm{U} \cdot \mathrm{mL}^{-1}$ penicillin, and $100 \mu \mathrm{g} \cdot \mathrm{mL}^{-1}$ streptomycin. Others cell lines were maintained in the nutrient DMEM similarly supplemented. All of these cells were grown at $37{ }^{\circ} \mathrm{C}$ in $5 \% \mathrm{CO}_{2}$ and humidified air atmosphere. The PBMC cells were separated by Histopaque-1077 (GE Healthcare), from whole blood of healthy, non-smoking donors who had not taken any drugs for at least 15 days prior to sampling donors. Thus, using the gradient centrifugation, the interface cells were washed with PBS (phosphate buffered saline), counted and resuspended in nutrient medium to the required cell concentration. The blood used to obtain PBMC cells were collected from the blood bank of the João Pessoa (Hemocentro), Paraíba, Brazil. Similar procedure was performed for obtaining PBMC/CML. These cells were collected from blood of patients recently diagnosed with CML and without being subject to treatment at this moment. The blood samples from patients PBMC/CML, were collected in the Napoleão Laureano Hospital, João Pessoa, Paraíba, Brazil. This study was approved by the Institutional Ethical Committee of Lauro Wanderley Hospital (CEP/HULW), from Federal University of Paraíba, protocol numbers, 378119 and 05878712.7.0000.5183.

\subsubsection{Cell Viability-MTT}

The cytotoxicity of molecules to cells was evaluated using the original enzymatic reduction of 3-(4,5-dimethylthiazol-2-yl)-2,5-diphenyltetrazolium bromide (MTT) assay to produce formazan crystals [33]. Cells were seeded at $3 \times 10^{4}$ or $5 \times 10^{4}$ cells per well in 96-well tissue culture plates. Cells were exposed to different concentrations of tetrahydropyrans substituted $\left(3-100 \mu \mathrm{mol} \cdot \mathrm{L}^{-1}\right)$ dissolved in the medium (three wells per concentration). After $24 \mathrm{~h}$ or $72 \mathrm{~h}$ of incubation, plates were centrifuged ( $500 \mathrm{~g}, 5 \mathrm{~min}$ ) and the supernatant was removed, followed by the addition of MTT solution $\left(0.5 \mathrm{mg} \cdot \mathrm{mL}^{-1}\right.$ in PBS) and incubation for $4 \mathrm{~h}$ at $37^{\circ} \mathrm{C}$. After $4 \mathrm{~h}$, the MTT formazan product was dissolved in SDS/ $\mathrm{HCl} 0.01 \mathrm{~mol} \cdot \mathrm{L}^{-1}$ and absorbance was measured at $570 \mathrm{~nm}$ in reader plate (Biotek, Winooski, VT, USA).

\section{Conclusions}

In this paper we present efficient syntheses of six new guanylhydrazone and aminoguanidine tetrahydropyran derivatives 2-7. We studied the cytotoxic activity of studied tetraydropyran compounds (2-7) in various cancer and normal cell lines; in K562 cells the cytotoxicity is mediated by cell-cycle arrest in the G1 phase. These results can address a promising starting point for furthering structural modifications in these compounds as a new class of anticancer compounds, leading to the possibility of target-directed drug design for cancer treatment.

Supplementary Materials: Supplementary materials can be accessed at: http://www.mdpi.com/1420-3049/21/ 6/671/s1.

Acknowledgments: This work has been supported by CNPq and CAPES. Chemistry Institute of Federal University of Goiás (UFG) by CHNS analysis.

Author Contributions: Fábio Pedrosa Lins Silva was responsible for the chemical experiments and analyzed the data; Bruna Braga Dantas and Gláucia Veríssimo Faheina Martins were responsible for pharmacology experiments Demétrius Antônio Machado de Araújo was responsible for pharmacology orientations; Mário Luiz Araújo de Almeida Vasconcellos was responsible for design of article, analyzed the data, Fabio P.L Silva orientation and wrote the paper.

Conflicts of Interest: The authors declare no conflict of interest.

\section{References}

1. Varmus, H. The New Era in Cancer Research. Science 2006, 312, 1162. [CrossRef] [PubMed]

2. Jemal, A.; Siegel, R.; Ward, E.; Hao, Y.; Xu, J.; Thun, M.J. Cancer statistics, 2009. CA Cancer J. Clin. 2009, 59, 225-249. [PubMed]

3. WHO Websites. Available online: http://www.who.int/cancer/en (accessed on 23 August 2015).

4. Cancer Trends Progress Report. Available online: http://progressreport.cancer.gov (accessed on 22 June 2015). 
5. Verweij, J.; Jonge, M.J.A. Achievements and future of chemotherapy. Eur. J. Cancer 2000, 36, $1479-1487$. [CrossRef]

6. Carrillo, R.; Leon, L.G.; Martín, T.; Martín, V.S.; Padrón, J.M. Synthesis and antiproliferative activity of $(2 R, 3 R)$-disubstituted tethahydropyrans. Bioorg. Med. Chem. Lett. 2006, 16, 6135-6138. [CrossRef] [PubMed]

7. Carrillo, R.; Leon, L.G.; Martín, T.; Martín, V.S.; Padrón, J.M. Synthesis and antiproliferative activity of $(2 R, 3 R)$-disubstituted tetrahydropyrans. Part 2: Effect of side chain homologation. Bioorg. Med. Chem. Lett. 2007, 17, 780. [CrossRef] [PubMed]

8. Singh, P.; Bhardwaj, A. Mono-, Di-, and Triaryl Substituted Tetrahydropyrans as Cyclooxygenase-2 and Tumor Growth Inhibitors. Synthesis and Biological Evaluation. J. Med. Chem. 2010, 53, 3707-3717. [PubMed]

9. Al-Tel, T.H. Design and synthesis of novel tetrahydro-2H-Pyrano[3,2-c]Pyridazin-3(6H)-one derivatives as potential anticancer agents. Eur. J. Med. Chem. 2010, 45, 5724-5731. [CrossRef] [PubMed]

10. Al-Tel, T.H. Design, synthesis and qualitative structure-activity evaluations of novel hexahydropyrano $[3,2-c][1,2]$ diazepin-3(4H)-one and tetrahydropyrano[3,2-b]pyrrol-2(1H)-one derivatives as anticancer agents. Eur. J. Med. Chem. 2010, 45, 4615-4621. [CrossRef] [PubMed]

11. Judd, W.R.; Slattum, P.M.; Hoang, K.C; Bhoite, L.; Valppu, L.; Alberts, G.; Brown, B.; Roth, B.; Ostanin, K.; Huang, L. Discovery and SAR of Methylated Tetrahydropyranyl Derivatives as Inhibitors of Isoprenylcysteine Carboxyl Methyltransferase (ICMT). J. Med. Chem. 2011, 54, 5031-5047. [CrossRef] [PubMed]

12. Vasconcellos, M.L.A.A.; Miranda, L.S.M. A reação de ciclização de Prins: Uma estratégia eficiente para síntese estereosseletiva de anéis tetraidropirânicos substituídos. Quím. Nova 2006, 29, 834-839. [CrossRef]

13. Ahmeda, N.; Konduru, K.; Ahmad, S.; Owais, M. Synthesis of flavonoids based novel tetrahydropyran conjugates (Prins products) and their antiproliferative activity against human cancer cell lines. Eur. J. Med. Chem. 2014, 75, 233-246. [CrossRef] [PubMed]

14. Miranda, L.S.M.; Marinho, B.G.; Leitão, S.G.; Matheus, M.E.; Fernandes, P.D.; Vasconcellos, M.L.A.A. $( \pm)$-cis-(6-Ethyl-tetrahydropyran-2-yl)-formic acid: A novel substance with antinociceptive properties. Bioorg. Med. Chem. Lett. 2004, 14, 1573-1575. [CrossRef] [PubMed]

15. Marinho, B.G.; Miranda, L.S.M.; Gomes, N.M.; Matheus, M.E.; Leitão, S.G.; Vasconcellos, M.L.A.A.; Fernandes, P.D. Antinociceptive action of $( \pm$ )-cis-(6-ethyl-tetrahydropyran-2-yl)-formic acid in mice. Eur. J. Pharmacol. 2006, 550, 47-53. [CrossRef] [PubMed]

16. Marinho, B.G.; Miranda, L.S.M.; Marinho, B.G.; Vasconcellos, M.L.A.A.; Matheus, M.E.; Pereira, V.L.P.; Fernandes, P.D. Antinociceptive activity of (-)-(2S,6S)-(6-ethyl-tetrahydropyran-2-yl)-formic acid on acute pain in mice. Behav. Pharmacol 2011, 22, 564-572. [CrossRef] [PubMed]

17. Capim, S.L.; Carneiro, P.H.P.; Castro, P.C.; Barros, M.R.M.; Marinho, B.G.; Vasconcellos, M.L.A.A. Design, Prins-cyclization reaction promoting diastereoselective synthesis of 10 new tetrahydropyran derivatives and in vivo antinociceptive evaluations. Eur. J. Med. Chem. 2012, 58, 1-11. [CrossRef] [PubMed]

18. Capim, S.L.; Gonçalves, G.M.; dos Santos, G.C.M.; Marinho, B.G.; Vasconcellos, M.L.A.A. High analgesic and anti-inflammatory in vivo activities of six new hybrids NSAIAs tetrahydropyran derivatives. Bioorg. Med. Chem. 2013, 21, 6003-6010. [CrossRef] [PubMed]

19. LaFrate, A.L.; Gunther, J.R.; Carlson, K.E.; Katzenellenbogen, J.A. Synthesis and biological evaluation of guanylhydrazone coactivator binding inhibitors for the estrogen receptor. Bioorg. Med. Chem. 2008, 16, 10075-10084. [CrossRef] [PubMed]

20. Pignatellol, R.; Panicol, A.; Mazzonez, P.; Pinizzotto, M.R.; Garozzo, A.; Furneri, P.M. Schiff bases of $N$-hydroxy- $N^{\prime}$-aminoguanidines as antiviral, antibacterial and anticancer agents. Eur. J. Med. Chem. 1994, 29, 781-785.

21. Basua, A.; Sinha, B.N.; Saiko, P.; Graser, G.; Szekeres, T. N-Hydroxy-N'-aminoguanidine as anti-cancer lead molecule: QSAR, synthesis and biological evaluation. Bioorg. Med. Chem. Lett. 2011, 21, 3324-3328.

22. Zhou, J.Y.; Jia, Y.; Sun, G.F.; Wu, S.H. Barbier-Type Allylation of Aldehydes and Ketones with Metallic Lead in Aqueous Media. Synth. Commun. 1997, 27, 1899-1906. [CrossRef]

23. Silva, F.P.L.; Sabino, J.R.; Martins, F.T.; Vasconcellos, M.L.A.A. Diastereoselective syntheses via Prins cyclization, crystal structures determination and theoretical studies of cis-2,6-diphenyl4-hydroxytetrahydropyran and analogues. J. Mol. Structure 2013, 1036, 478-487. [CrossRef] 
24. El-Azab, A.; Al-Omar, M.A.; Abdzel-Aziz, A.A.M.; Abdzel-Aziz, N.I.; El-Sayed, M.A.A.; Aleisa, A.M.; Sayed-Ahmed, M.M.; Abdel-Hamide, S.G. Design, synthesis and biological evaluation of novel quinazoline derivatives as potential antitumor agents: Molecular docking study. Eur. J. Med. Chem. 2010, 45, 4188-4198. [CrossRef] [PubMed]

25. Rowley, J.D. A New Consistent Chromosomal Abnormality in Chronic Myelogenous Leukaemia identified by Quinacrine Fluorescence and Giemsa Staining. Nature 1973, 243, 290-293. [CrossRef] [PubMed]

26. Baccarani, M.; Cortes, J.; Pane, F.; Niederwieser, D.; Saglio, G.; Apperley, J.; Cervantes, F.; Deininger, M.; Gratwohl, A.; Guilhot, F.; et al. Chronic Myeloid Leukemia: An Update of Concepts and Management Recommendations of European LeukemiaNet. J. Clin. Oncol. 2009, 27, 6041-6051. [CrossRef] [PubMed]

27. Slevin, M.L. The clinical pharmacology of etoposide. Cancer 1991, 67, 319-329. [CrossRef]

28. Saleh, E.M. Inhibition of topoisomerase II $\alpha$ sensitizes FaDu cells to ionizing radiation by diminishing DNA repair. Tumour Biol. 2015, 10, 8985-8992. [CrossRef] [PubMed]

29. Torres, F.Q.J.; Díaz, J.G.; Carmona, A.J.; Estévez, F. Trifolin acetate-induced cell death in human leukemia cells is dependent on caspase-6 and activates the MAPK pathway. Apoptosis 2008, 13, 716-728. [CrossRef] [PubMed]

30. Roos, W.P.; Thomas, A.D.; Kaina, B. DNA damage and the balance between survival and death in cancer biology. Nat. Rev. Cancer 2016, 16, 20-33. [CrossRef] [PubMed]

31. Szücová, L.; Lukáš, S.; Dolez, K.; Zatloukal, M.; Greplová, J.; Galuszka, P.; Kryštof, V.; Voller, J.; Popa, I.; Massino, F.J.; et al. Synthesis, characterization and biological activity of ring-substituted 6-benzylamino9-tetrahydropyran-2-yl and 9-tetrahydrofuran-2-ylpurine derivatives. Bioorg. Med. Chem. 2009, 17, 1938-1947. [CrossRef] [PubMed]

32. Pedram, B.; van Oeveren, A.; Mais, D.E.; Marschke, K.B. A Tissue-Selective Nonsteroidal Progesterone Receptor Modulator: 7,9-Difluoro-5-(3-methylcyclohex-2-enyl)-2,2,4-trimethyl-1,2-dihydrochromeno[3,4-f] quinoline. J. Med. Chem. 2008, 51, 3696-3999. [CrossRef] [PubMed]

33. Mosmann, T. Rapid colorimetric assay for cellular growth and survival: Application to proliferation and cytotoxicity assays. J. Imunol. Methods 1983, 65, 55-63. [CrossRef]

Sample Availability: Samples of the compounds 2-7 are available from the authors.

(c) 2016 by the authors; licensee MDPI, Basel, Switzerland. This article is an open access article distributed under the terms and conditions of the Creative Commons Attribution (CC-BY) license (http://creativecommons.org/licenses/by/4.0/). 